\title{
Comparison of two-step transient evoked otoacoustic emissions (TEOAE) and automated auditory brainstem response (AABR) for universal newborn hearing screening programs
}

\author{
J.I. Benito-Orejas ${ }^{a, *}$, B. Ramírez ${ }^{a}$, D. Morais ${ }^{a}$, \\ A. Almaraz ${ }^{b}$, J.L. Fernández-Calvo ${ }^{c}$ \\ ${ }^{a}$ ENT Department, Hospital Clínico Universitario, Valladolid, Spain \\ ${ }^{\mathrm{b}}$ Research Support Unit, Hospital Clínico Universitario, Valladolid, Spain \\ c Pediatrics Department, Hospital Clínico Universitario, Valladolid, Spain
}

Received 28 January 2008; received in revised form 20 April 2008; accepted 22 April 2008 Available online 12 June 2008

This project is dedicated to Dr. María Angeles Condado, "in memoriam”.

\section{KEYWORDS}

Universal newborn hearing screening; Screening; Hearing; Transient evoked otoacoustic emissions; Automated auditory brainstem response

\begin{abstract}
Summary
Objective: Both transitory auditory otoemissions (TEOAE) and automated auditory brainstem responses (AABR) are considered adequate methods for universal hearing screening. The goal of this study was to compare the results obtained with each device, applying the same screening procedure.

Materials and methods: From 2001 to 2003, all the newborns in our health area (2454 infants) were evaluated with TEOAE (ILO92, otodynamics) and all those born from 2004 to 2006 (3117) were evaluated with AABR (AccuScreen, Fischer-Zoth). The population studied included all well newborns and those admitted to neonatal intensive care units (NICU). The first screening was normally undertaken with well babies during the first $48 \mathrm{~h}$ of life, before hospital discharge. Infants referred from this first step underwent a second screening after hospital discharge, before they were a month old.

Results: The results from each study group were compared and analyzed for significant differences. TEOAE screening yielded $10.2 \%$ fail results from the first screening step; AABR gave 2.6\%. In the second screening step, $2 \%$ of the newborns screened with TEOAE were referred, whereas $0.32 \%$ of those screened with AABR were referred. These differences are statistically significant.
\end{abstract}

\footnotetext{
* Corresponding author at: Avda. Salamanca 16-1A, 47014 Valladolid, Spain. Tel.: +34 983338797.

E-mail address: jibenito@ono.com (J.I. Benito-Orejas).
} 
Conclusions: Although AABR screening tests involve a slightly higher cost in time and money than TEOAE, the results obtained compensate this difference. AABR gives fewer false positives and a lower referral rate; the percent of infants lost during follow-up is consequently smaller. Therefore, in our environment, universal newborn auditory screening with AABR is more effective than that with TEOAE.

(C) 2008 Elsevier Ireland Ltd. All rights reserved.

\section{Introduction}

The goal of newborn auditory screening is to identify accurately infants with significant hearing impairment in the most rapid and cost-effective way [1]. Unfortunately, the ideal hearing-screening test for children has yet to be defined [2] and local circumstances may make variations necessary. However, a low false-positive rate is central to the success of a pre-discharge neonatal hearing-screening program. The multi-site study by Norton et al. [3,4], funded by the National Institute on Deafness and Other Communication Disorders (NIDCD), compared the effectiveness of transient evoked otoacoustic emissions (TEOAE), distortion product otoacoustic emissions (DPOAE) and auditory brainstem response (ABR). They concluded that, taking the visual reinforcement audiometry (VRA) at 8-12 months corrected age as a reference, all three methods serve equally well for newborn hearing screening. None of these methods measure hearing loss; their goal is objectively evaluating adequate hearing function [5]. TEOAE and ABR equipment is constantly evolving and becoming more and more automated, with different screening programs being established in a single phase or several phases for each method. Determining which of them is most effective is therefore of great interest.

TEOAE tests are generally thought to be easier to administer [6] (as scalp electrodes are not required) and faster [5,7], offering referral rates for the first screening that range from $6 \%$ to $12 \%$ [8-12]. These rates can even increase in preterm infants [13-15] and newborns younger than $48 \mathrm{~h}$ up to $60 \%$ [16-19]. $A A B R$ tests yield lower first-screening referral rates $(1-4 \%)[7-9,11,20-22]$. If the program is a two-step screen, the TEOAE referral rate following the second test can drop to $6-7 \%[10,12,23-26]$. However, AABR seems to give fewer false positives, which range from $1 \%$ to $2 \%$ after the second screening step $[5,11,18,20,22]$.

The goal of this study was to compare the results of a two-step screening process with both TEOAE and $A A B R$ in a similar population of newborns.

\section{Materials and methods}

Our Autonomous Community, Castilla y León, consists of 9 provinces and has 14 public hospitals equipped with Maternity Service, including our Hospital Clínico in Valladolid. Political consensus facilitated the preparation, with the agreement of the professionals involved, of a set of protocols approved as the "Program for Early Detection of and Integrated Attention to Infant Hearing Loss in Castilla y León" [27]. The program was implemented in September 2004, and a hand-held Automatic Auditory Brainstem Response (AABR) device was chosen for performing the hearing screening, considering that $A A B R$ could be more efficient in our environment than TEOAE.

Given that screening of all newborns at the Hospital Clínico in Valladolid with TEOAE started in March 2001, it is possible to compare the results obtained during the first 2 years this protocol was applied (March 2001-February 2003) against those from the first 2 years of AABR tests (September 2004-August 2006). Statistical treatment was performed in collaboration with the "Research Support Unit" at our hospital, using "EPIDAT" statistical software. Results were compared using chi-squared test, $p<0.05$ being considered significant.

The study was reviewed and approved by the scientific research committee of the Hospital Clínico in Valladolid.

\subsection{Subjects}

The population evaluated included all newborns from the periods indicated (well babies and those with factors of risk for hearing loss, including newborns admitted to neonatal intensive care units (NICU)). A total of 5571 newborns were available for screening; 2454 were screened with TEOAE (3.3\% presented risk factors and $13.5 \%$ came from NICUs) and 3117 with AABR (4.6\% with risk factors and $14.8 \%$ from NICUs).

In our hospital, well babies are discharged from 24 to $48 \mathrm{~h}$ after delivery (except for caesarean births, where discharge is a few days later). The initial screening test is performed during this time. Newborns in NICUs are tested on the day they are discharged.

The screenings were performed at the mothers' bedsides under usual postnatal ward conditions, with the infants in their cots, held in their mothers' arms, or being nursed at the breast. Informed consent was received from the mother before the 
hearing screen. NICU infants were screened in our ENT Service.

All auditory screenings were done by a nurse in the Otorhinolaryngology (ENT) Service. (There were two nurses, who took turns to cover vacation periods.) Auditory screening was not done at the weekend or on holidays, so if a newborn was discharged without being screened, an appointment for a few days later was given. Outpatient babies (infants who were referred from in-hospital screening and those who were not accessed or successfully screened before discharge) were screened in our ENT Service.

The same nurses who began and perfected their learning curve with TEOAE screening also had to be trained in AABR testing, given that the two procedures are different. All the data obtained in each stage were recorded in a specific computer database.

Parents were given the results of the hearing screening prior to discharge in written and verbal format. They also received a guide with normal speech and language development indicators, so they would be able to evaluate and watch over future language evolution.

\subsection{Hearing-screening equipment}

Newborns were screened for hearing loss with an accepted physiologic measure of auditory function $[19,28]$ : automated auditory brainstem responses (AABR) or transient evoked otoacoustic emissions (TEOAE).

TEOAE are elicited by click stimulus delivered by a probe transducer in the external ear canal. The emission or "echo" from outer hair cells of the inner ear is recorded by a microphone in an external ear probe assembly [8]. TEOAE presence implies integrity of sound transmission through the outer and middle ear structures and functional integrity of the outer hair cells.

The equipment used for TEOAE screening was an IL092 System (Otodynamics Ltd., Hatfield, UK), using the Quickscreen program (which reduces test time and minimizes the effects of low-frequency noise interference on the detection of TEOAE), and applying the following validation criteria [12]:

- Trial Validation: background noise $<45 \mathrm{~dB}$ sound pressure level (SPL); a minimum of 50 quiet sweeps collected; stimulus intensity $<86 \mathrm{~dB}$ SPL; stimulus stability $\geq 75 \%$.

- Response Validation: whole wave reproducibility $\geq 70 \%$; global signal-to-noise ratio $\geq 10 \mathrm{~dB}$ SPL; three additional signal-to-noise ratios met $(1500 \mathrm{~Hz} \geq 50 \%$ or $\geq 3 \mathrm{~dB}$, and 2200-3000$4000 \mathrm{~Hz} \geq 70 \%$ or $\geq 6 \mathrm{~dB}$ ). If a trial was technically adequate and these criteria were achieved, the trial was a "pass".

Auditory brainstem responses imply functional integrity from the eighth nerve through the auditory brainstem.

AABR were obtained using an AccuScreen Pro ABR from Fischer-Zoth, Germany (Madsen-GN Otometrics, Taastrup, Denmark); the device was portable and automatic ("pass"-_refer"), the result requiring no interpretation.

To perform $A A B R$, three disposable adhesive gel electrodes were placed on the infants (the noninverting electrode on the upper forehead, the inverting or referent electrode on the nape of the neck and a ground electrode on the shoulder). Impedances accepted, between any two sites, were always lower than $12 \mathrm{k} \Omega$. The stimulus consisted of $100 \mu \mathrm{s}$ clicks of alternating polarity at a frequency of approximately $55 \mathrm{~Hz}$ and an intensity of $35 \mathrm{~dB} \mathrm{nHL}$ (hearing level click stimulus referenced to normal hearing in adults). The clicks were sent through cushioned coupler earphones attached around the newborn's ears, so a probe did not interfere with the external auditory canal; in addition, the earphones isolated the newborn from environmental noise. Data was shown in an 18-ms window post-stimulus, applying a binomial statistical evaluation algorithm over the sinuous pattern signal, which offered a confidence level of $>\mathbf{9 9 . 5 \%}$ for the response. The waveform generated from the brainstem was compared with a template derived from normally hearing infants. The equipment screen showed a graph that indicated test progress, EEG level and ABR detection probability. The equipment had a builtin rejection device for myogenetic, electrical and environmental noise interference that stopped the screening when testing conditions would preclude adequate testing. Based on the results, a "pass" or "refer" was generated after presentation of up to 15,000 stimuli.

\subsection{Hearing-screen protocols}

The methodology used in the detection of newborn hearing loss has already been described [12]. Summarizing briefly, it consists of a first screening step, which is repeated during the first month of life if the newborns is referred in one or both ears. When the "captive period" and circumstances allow, the newborns referred at the initial screening are rescreened before hospital discharge (the "pass" or "refer" result is still considered as "initial screening step"). Infants referred from the second screening enter formal audiological assessment. 
The physiologic techniques for screening hearing were incorporated into two different screening protocols:

Protocol 1 (March 2001-February 2003):

- TEOAE screening was performed using an ILO 92 system.

Infants with "refer" results in one or both ears underwent repeat TEOAE during the first month of life.

Protocol 2 (September 2004-August 2006):

- AABR screening was performed using a thirdgeneration AABR device (AccuScreen ProABR, Fischer-Zoth).

Infants with "refer" results in one or both ears underwent repeat $A A B R$ during the first month of life.

\section{Results}

Table 1 shows the comparison of the results obtained from Protocol 1 (two-step TEOAE screening) and Protocol 2 (two-step AABR screening). The differences between both universal hearing-screening protocols are statistically significant $(p<0.05)$ in all the variables chosen, except for the number of hearing loss cases diagnosed with each procedure.

The population studied with TEOAE consisted of 2454 newborns during the period from March 2001 to February 2003. That studied with AABR was 3117 newborns, likewise over 2 years (September 2004 to August 2006). The increase in the number of infants in the second period was due not only to a rise in birth rate, but to the fact that all newborns born in private health centers with a maternity service affiliated with our hospital were included in the screening.

The main results can be summarized as follows: with TEOAE, $10.2 \%$ of the newborns screened were referred at the first screening stage, in contrast with $2.6 \%$ using AABR; $2 \%$ of newborns were referred at the second stage with TEOAE, against $0.32 \%$ of those screened with AABR; $5.2 \%$ of newborns screened with TEOAE (comprising the "refer" $(2 \%)$ and the "pass" with risk factors (3.2\%)) were referred for ENT diagnosis, while only $0.32 \%$ of those screened with AABR were referred (only the "refer" group).

Based on the $V$ wave thresholds obtained with $A A B R$, we classified the hearing losses as mild (40$50 \mathrm{~dB} \mathrm{nHL})$, moderate-severe $(60-90 \mathrm{~dB} \mathrm{nHL})$ and profound ( $>90 \mathrm{~dB} \mathrm{nHL})$. Through TEOAE hearing screening, we diagnosed 13 children with permanent hearing loss ( 5 profound bilateral hearing loss, 3 moderate-severe bilateral, 1 case of moderatesevere hearing loss in one ear and mild in the other, 2 bilateral mild and 2 unilateral mild hearing loss cases), of which $54 \%(7 / 13)$ had associated risk factors for hearing loss. The AABR procedure yielded six children with permanent hearing loss (one profound bilateral hearing loss case, two bilateral moderate-severe, one case of moderate-severe hearing loss in one ear and mild in the other, and two mild bilateral hearing loss cases), of which $33 \%$ $(2 / 6)$ had associated risk factors.

Table 1 Results of two-stage newborn hearing screening with TEOAE and AABR

\begin{tabular}{|c|c|c|c|c|}
\hline & \multicolumn{2}{|l|}{ TEOAE } & \multicolumn{2}{|l|}{ AABR } \\
\hline & $N$ (infants) & $\%$ & $N$ (infants) & $\%$ \\
\hline Number of newborns & 2482 & & 3129 & \\
\hline No-show for first screening & $28 / 2482$ & 1.2 & $12 / 2858^{a}$ & 0.5 \\
\hline Number explored & 2454 & 98.8 & $2846^{a}$ & 99.5 \\
\hline "Fail" first screening & $250 / 2454$ & 10.2 & $81 / 3117$ & 2.6 \\
\hline No-show for second screening & $15 / 250$ & 6 & $1 / 81$ & 1.2 \\
\hline "Fail" second screening & $49 / 2439$ & 2 & $10 / 3116$ & 0.32 \\
\hline Referred for ENT diagnosis & $126 / 2439$ & 5.2 & $10 / 3116$ & 0.32 \\
\hline No-show for diagnosis & $32 / 2439$ & 1.3 & $2 / 3116$ & 0.06 \\
\hline Hearing loss & $13 / 2439$ & 0.54 & $6 / 3114$ & 0.19 \\
\hline \multicolumn{5}{|l|}{ False-positive rates } \\
\hline First screening & $237 / 2454$ & 9.7 & $75 / 3117$ & 2.4 \\
\hline Second screening & $36 / 2439$ & 1.47 & $2 / 3114$ & 0.064 \\
\hline \multicolumn{5}{|c|}{ Positive hearing loss predictive value } \\
\hline First screening & $13 / 250 \times 100$ & 5.2 & $6 / 81 \times 100$ & 7.4 \\
\hline Second screening & $13 / 49 \times 100$ & 26.5 & $6 / 8 \times 100$ & 75 \\
\hline
\end{tabular}


The rate of false positives for the first screening phase was $9.7 \%$ with TEOAE and $2.4 \%$ with AABR. For the second stage, the false positive rate was $1.5 \%$ with TEOAE and $0.06 \%$ with AABR. The hearing loss predictive value following the two screening steps was $26.5 \%$ for TEOAE and $75 \%$ for AABR.

With TEOAE, $15.3 \%$ of the newborns had to be screened after hospital discharge (outside of the "captive period") because the discharge coincided with holidays or weekends (not covered by screening). With AABR, only $6.4 \%$ were screened after hospital discharge because the test could be performed from a few hours following birth. These differences were the reason why the percent of newborns not appearing for the first screening after discharge was $1.13 \%$ with TEOAE, in contrast to $0.42 \%$ with AABR. Likewise, $6 \%$ of newborns who were referred at the first screening with TEOAE did not appear for the second screening, in comparison with $1.2 \%$ of those not appearing with $A A B R$ screening.

Our results were consistent with those reported by other researchers [8-26].

\section{Discussion}

Clinical OEA application made it possible to universalize newborn hearing screening because it provided a simple, quick, effective and non-invasive method for evaluating cochlear function [29-31]. However, the later ABR and TEOAE automation, even in the same equipment, made it easier to evaluate different protocols, where the combination of one or the other test in one or two stages achieved the greatest effectiveness [6]. In 1993, the National Institute of Health (NIH) [32] recommended an initial TEOAE test and, if the result was "refer", an immediate ABR test as the preferred screening model $[2,7,11,33,34]$. Several later studies $[9,18]$ criticized this model, because higher referral rates were obtained following the second test than if only one test with AABR had been performed. Motives for these results included the fact that if the AABR is done just after the TEOAE, the possibilities that the newborn will awake or that its noise level will increase are greater, thus impeding $A A B R$ results [6]. In addition, upon introducing a probe in the external auditory canal for the TEOAE, it is easy to drag wax into this canal and obstruct it, which makes obtaining the AABR more difficult $[6,18,35]$.

In our hearing-screening protocol, we used two steps with TEOAE or two steps with AABR [18,20$22,36]$. However, we do feel, in agreement with Clemens et al. [21,37], that retesting within a short interval (i.e., hours) is effective in reducing false positives. This is logical given that ambient sound and myogenetic interference are among the most commonly implicated factors in failed screening. A change of the infant's position or activity or a change in the location of the test will frequently change the screening result from fail to pass $[36,38]$. When a newborn failed the test in the maternity ward, we tried to repeat it, transferring the child to the ENT Service.

The functional design of the two units used in our study is current. Although with exceptions, most of the published data regarding TEOAE have been obtained through the use of hardware and software supplied by Otodynamics Ltd. and known as ILO. The suitability of the AABR equipment used for the test has been evaluated previously, comparing it with other third-generation AABR devices [5], and using it in hearing screening [6].

Tests were performed on well babies in the maternity ward in their mothers' rooms. This frequently helped to calm the babies, reducing myogenetically caused noise. With $A A B R$, using an ear probe introduced in the auditory canal offers results similar to those from coupler earphones [5]; we prefer coupler earphones because using this system isolates the newborn from maternity ward noise. Even accepting that this way of working is a bit more uncomfortable than transferring the newborn to an isolated room $[5,18,20]$, it results in more normality, trust and greater acceptance of the test on the part of the mothers.

Our results compared hearing screening with TEOAE and with AABR devices (Table 1). It can be seen that $A A B R$ reduces the rate of false positives significantly with respect to TEOAE; this reduces the number of infants that have to be retested outside the "captive period" and, consequently, the number who do not appear for retesting $[20,24,31,32,36,39,40]$.

Generally, the recommended age to begin screening with TEOAE is $48 \mathrm{~h}$ [16-19] because the presence of debris and vernix in the external meatus of the newborn is normally significantly reduced by the second day of life. However, due to the developing trend of shorter post-delivery hospital stays, we screen well newborns younger than $48 \mathrm{~h}$. This increases the number of false positives. The use of $A A B R$ is more time efficient than using TEOAE because of the significantly increased pass rates $[17,18]$.

On the other hand, differences in the method of OAE analysis might account for the difference in the $\mathrm{OAE}$ failure rate [7]. The more stringent the criteria, the higher the false-positive rate will be [8]. The numerical validation indexes of TEOAE response 
that we applied are similar to those recommended by the National Consortium on Newborn Hearing Screening [41] and those applied by Norton et al. [3] in their multicenter study. The criteria are fairly conservative and may refer more neonates with normal auditory status that if a more liberal pass/ refer system is adopted $[8,23,24,35]$. However, the study by Gabbard et al. [17] shows that this difference is barely significant.

The TEOAE results for failing the first screening $(10.2 \%)$ and failing the second screening $(2 \%)$ are similar to those of some authors $[10,11,19]$, but better than those of others $[1,16-18,35,38,40]$.

The percent of infants referred for diagnosis also drops considerably with AABR; this reduces expense, anxiety for families [20-22,25, 30,37, 40,42,43] and traveling to the diagnosis center, sometimes quite far from the families' homes. With TEOAE, infants who fail the second screening $(2 \%)$ and those who pass but have risk factors for hearing loss (3.2\%) are referred for diagnosis. AABR testing, as it evaluates the functionality of the auditory pathway, also facilitates diagnosing cases of auditory neuropathy that present normal TEOAE results $[6,40,44]$. This avoids having to do $A B R$ on infants with risk factors who "pass" the AABR, which is especially important in centers that depend on another reference center for performing the $A B R$. In these cases, the rate of infants not appearing is very high $[36,39]$, because (in addition to the problem of traveling) the parents do not understand the reason why they have to return, given that their child have "passed" the TEOAE screening test and apparently present a normal response to sound.

With marginalized groups or, in our environment, with the gypsy ethnic group, reducing the number of infants that have to be retested outside the period of captivity is very important: first, because they present a higher incidence of congenital hearing loss [12], perhaps due to frequent kinship relationships; and second, because follow-up is especially difficult, given their marginalized situation. Fifty percent of the infants who failed the second $A A B R$ screening test and the two cases in which the diagnostic stage was rejected belonged to these groups. It is therefore essential to take advantage of the captivity period as much as possible, and to repeat the screening test as often as needed to achieve reliable results. If these infants do not pass the test in the end, precautions that ensure adequate follow-up will then be taken.

Excluding the two infants mentioned above, we can say that there was really no false positive in the remaining eight newborns that did not pass the second AABR screening. The two infants for whom a permanent hearing loss was not confirmed pre- sented bilateral serous otitis media during the screening tests; the cause of their "fail" was thus the result of a transitory transmission hearing loss $[4,20]$. However, mechanical factors in the middle ear, basilar membrane immaturity or development, increased myelination in the auditory pathway or transitory neurological anomalies could be the cause of false positives with AABR $[45,46]$.

We detected a greater number of hearing loss cases in the hearing-screening protocol with TEOAE than in that of AABR. However, given the low prevalence of congenital hearing loss and the number of newborns studied, these differences are not statistically significant. Nevertheless, these results could mean that TEOAE, although it is less specific than $A A B R$, is more sensitive. In previous studies $[13,20,47,48]$, AABR sensitivity (compared with the conventional ABR method) oscillated between $90 \%$ and $100 \%$. Accepting the pass/no pass criteria of $35 \mathrm{~dB} H \mathrm{HL}$ [28], some infants will pass the AABR hearing screening in spite of having a certain degree of mild permanent hearing loss $[28,49]$. If we want to identify all the degrees of hearing loss, we can lower the AABR screening level, but that would increase the false positives considerably; in addition, the costs associated would be so high that the program, probably, could not survive. Choosing the criteria that allow infants with "moderate or greater hearing loss" ( $>35 \mathrm{~dB} \mathrm{HL}$ ) to be detected, the false positives and costs can be reduced [50].

$A B R$ is very precise in determining the average threshold of frequencies at $2000-4000 \mathrm{~Hz}$. However, its sensitivity is quite low under $1000 \mathrm{~Hz}$ and above $4000 \mathrm{~Hz}$, so false negatives could appear in hearing losses of unusual configurations [51]. Likewise, differences in the size of the external auditory canal and in the placement and type of earphone can produce small differences in the stimulus, giving rise to false negatives in mild hearing losses [49].

The Norton NIH study data [3] would suggest that OAEs are no more sensitive to mild hearing loss than ABR [49].

Although TEOAE hearing screening was easier and more comfortable to perform than that of AABR [5$7,19,22,26]$, the differences are becoming less and less important. As new generations of AABR devices are developed, administration time is decreasing. It is true that screening test time varies depending on the child's hearing, the presence of partial obstructions in the external auditory canal or the middle ear, and on test conditions. However, the average time needed to carry out automated $A B R$ testing ranges from 8 to $15 \mathrm{~min}[2,5,9,16-18,20,22,26,36,38-40]$, and conventional TEOAE tests take from 2 to $13 \mathrm{~min}$ $[1,2,9,10,16,17]$. We calculate, without exact measurements, that each test took an average of $15 \mathrm{~min}$ 
using the specified $A A B R$ equipment and about $10 \mathrm{~min}$ using that of TEOAE.

Studies calculating and comparing the expenses involved in two-step TEOAE testing with twostep AABR testing indicate they are analogous $[9,18,38,40]$. It is true that disposable supply costs are practically negligible with TEOAE and approximately $€ 7$ per child with our AABR equipment (the price of earphones and electrodes) [5]. However, the lower false positive rates achieved with AABR and, above all, the lower referral rate with this test make the total pre- and post-discharge costs for the two tests similar $[9,26]$. In our hospital, integrated in the public health network, the personnel that administered the screening tests and the schedule used to perform them was the same in both procedures. Therefore, using AABR, tangible costs increased by $€ 7$ per child, but it is very difficult to calculate the intangible cost savings (anxiety, trips back and forth, time lost from work, etc.) that the lower number of false positives means for the parents.

As its name indicates, our "Program for Early Detection of and Integrated Attention to Infant Hearing Loss in Castilla y León" contemplates not only newborn hearing screening, but also the procedures for diagnosis and multidisciplinary attention. These are fundamental in a program whose global goal is taking care of hearing [43]. An essential part of this attention is the support given by the primary health care pediatrician $[8,29,52]$. In the periodic newborn controls, this professional verifies the performance of screening tests, plans follow-up for children with risk factors and evaluates the appropriate diagnostic and therapeutic attention in hearing loss, whether confirmed or still to be confirmed. In addition, given that newborn auditory screening does not exclude the appearance of late onset hearing loss [29], pediatricians have the responsibility of an early diagnosis when faced with the slightest suspect, on their part or that of family members, of hearing problems.

\section{Conclusions}

The two-step screening test with AABR does not involve excessive costs in time or money with respect to TEOAE testing. We therefore feel it is the preferred current method for universal newborn hearing screening in our circumstances, as AABR testing gives a lower rate of false positives. When referral rates at hospital discharge decrease, the number of infants not appearing for the second stage of screening and the referral rates for diagnosis drop. This in turn lowers intangible costs including transportation fees, time off during follow-up and parental anxiety from the waiting period. Furthermore, AABR measurements reflect the status of the peripheral auditory system and (in contrast to those of OAE) permit detection of neural conduction disorders. Finally, it must be remembered that the identification of mild hearing loss in the neonatal period is not straightforward, that no screening test performs perfectly and that the screening program will never identify late-onset hearing loss. Therefore, regardless of the results of the infant hearing screening, the parents, medical and nursing personnel, and all other professionals who have opportunity to observe the child should pay attention to risk factors and to the behavioral signs of a possible change in the child's hearing status or language development.

\section{Acknowledgements}

The authors wish to thank the ENT nurses Esther Juárez and Mamen Vaquero, as responsible for our newborn hearing screening, for their constant efforts and support.

\section{References}

[1] K.J. Doyle, B. Burggraaff, S. Fujikawa, J. Kim, Newborn hearing screening by otoacoustic emissions and automated auditory brainstem response, Int. J. Pediatr. Otorhinolaryngol. 41 (1997) 111-119.

[2] M. Hahn, A. Lamprecht-Dinnesen, A. Heinecke, S. Hartmann, S. Bülbül, G. Schröder, et al., Hearing screening in healthy newborns: feasibility of different methods with regard to test time, Int. J. Pediatr. Otorhinolaryngol. 51 (1999) 83-89.

[3] S.J. Norton, M.P. Gorga, J.E. Widen, R.C. Folsom, Y. Sininger, B. Cone-Wesson, et al., Identification of neonatal hearing impairment: evaluation of transient evoked otoacoustic emission, distortion product otoacoustic emission and auditory brain stem response test performance, Ear Hear. 21 (2000) 508-528.

[4] S.J. Norton, M.P. Gorga, J.E. Widen, R.C. Folsom, Y. Sininger, B. Cone-Wesson, et al., Identification of neonatal hearing impairment: summary and recommendations, Ear Hear. 21 (2000) 529-535.

[5] S. Meier, O. Narabayashi, R. Probst, N. Schmuziger, Comparison of currently available devices designed for newborn hearing screening using automated auditory brainstem and/ or otoacoustic emission measurements, Int. J. Pediatr. Otorhinolaryngol. 68 (2004) 927-934.

[6] S.G. Korres, D.G. Balatsouras, C. Lyra, D. Kandiloros, E. Ferekidis, A comparison of automated auditory brainstem responses and transiently evoked otoacoustic emissions for universal newborn hearing screening, Med. Sci. Monit. 12 (6) (2006) CR260-CR263.

[7] C.R. Kennedy, L. Kimm, D. Cafarelli, P.I.P. Evans, M. Hunter, S. Lenton, R.D. Thornton, Otoacoustic emissions and audi- 
tory brainstem responses in the newborn, Arch. Dis. Child. 66 (1991) 1124-1129.

[8] T. Finitzo, K. Albright, J. O'Neal, The newborn with hearing loss: detection in the nursery, Pediatrics 102 (6) (1998) $1452-1460$.

[9] B.R. Vohr, W. Oh, E.J. Stewart, J.D. Bentkover, S. Gabbard, J. Lemons, et al., Comparison of costs and referral rates of 3 universal newborn hearing screening protocols, J. Pediatr. 139 (2) (2001) 238-244.

[10] H.C. Lin, M.T. Shu, K.C. Chang, S.M. Bruna, A universal newborn hearing screening program in Taiwan, Int. J. Pediatr. Otorhinolaryngol. 63 (2002) 209-218.

[11] A.L. Mehl, V. Thomson, The Colorado Newborn Hearing Screening Project, 1992-1999: on the threshold of effective population-based universal newborn hearing screening, Pediatrics 109 (2002) 1-8.

[12] R. Martínez, J.I. Benito, Condado MaAA, D. Morais, J.L. Fernández Calvo, Resultados de aplicar durante 1 año un protocolo universal de detección precoz de la hipoacusia en neonatos, Acta Otorrinolaringol. Esp. 54 (2003) 309-315.

[13] H.L.M. van Straaten, M.E. Groote, A.M. Oudesluys-Murphy, Evaluation of an automated auditory brainstem response infant hearing screening method in at risk neonates, Eur. J. Pediatr. 155 (1996) 702-705.

[14] H.L.M. van Straaten, C.H. Tibosch, C. Dorrepaal, F.W. Dekker, J.H. Kok, Efficacy of automated auditory brainstem response hearing screening in very preterm newborns, J. Pediatr. 138 (5) (2001) 674-678.

[15] H.L.M. van Straaten, E.T.M. Hille, J.H. Kok, P.H. Verkerk, N.I.C.U. the Dutch NICU Neonatal Hearing Screening Working Group, Implementation of a nation-wide automated auditory brainstem response hearing screening programme in neonatal intensive care units, Acta Paediatr. 92 (2003) $332-338$.

[16] K.J. Doyle, S. Fujikawa, P. Rogers, E. Newman, Comparison of newborn hearing screening by transient otoacoustic emissions and auditory brainstem response using ALGO-2 ${ }^{\mathbb{R}}$, Int. J. Pediatr. Otorhinolaryngol. 43 (1998) 207-211.

[17] S.A. Gabbard, J.L. Northern, C. Yoshinaga-Itano, Hearing screening in newborns under 24 hours of age, Semin. Hear. 20 (1999) 291-305.

[18] P. Clarke, M. Iqbal, S. Mitchell, A comparison of transientevoked otoacoustic emissions and automated auditory brainstem responses for pre-discharge neonatal hearing screening, Int. J. Audiol. 42 (2003) 443-447.

[19] American Academy of Pediatrics, Newborn and infant hearing loss: detection and intervention, Pediatrics 103 (1999) 527-530.

[20] J.A. Mason, K.R. Herrmann, Universal infant hearing screening by automated auditory brainstem response measurement, Pediatrics 101 (2) (1998) 221-228.

[21] C.J. Clemens, S.A. Davis, Minimizing false-positives in universal newborn hearing screening: a simple solution, Pediatrics 107 (3) (2001) e29.

[22] S. Iwasaki, Y. Hayashi, A. Seki, M. Nagura, Y. Hashimoto, G. Oshima, T. Hoshino, A model of two-stage newborn hearing screening with automated auditory brainstem response, Int. J. Pediatr. Otorhinolaryngol. 67 (2003) 1099-1104.

[23] A.B. Maxon, K.R. White, T.R. Behrens, B.R. Vohr, Referral rates and cost efficiency in a universal newborn hearing screening program using transient evoked otoacoustic emissions, J. Am. Acad. Audiol. 6 (1995) 271-277.

[24] G.D. Ceulaer, K. Daemers, K. van Driessche, et al., Neonatal hearing screening with transient evoked otoacoustic emissions: a learning curve, Audiology 38 (1999) 296-302.

[25] M. Owen, M. Webb, K. Evans, Community based universal neonatal hearing screening by health visitors using otoa- coustic emissions, Arch. Dis. Child. Fetal Neonatal Ed. 84 (2001) F157-F162.

[26] H.C. Lin, M.T. Shu, K.S. Lee, G.M. Ho, T.Y. Fu, S. Bruna, G. Lin, Comparison of hearing screening programs between one step with transient evoked otoacoustic emissions (TEOAE) and two steps with TEOAE and automated auditory brainstem response, Laryngoscope 115 (11) (2005) 19571962.

[27] Junta de Castilla y León, Consejería de Sanidad, Programa de detección precoz y atención integral de la hipoacusia infantil, 2004, $78 \mathrm{pp}$.

[28] Joint Committee on Infant Hearing (Special Article), Year 2000 position statement: principles and guidelines for early hearing detection and intervention programs, Pediatrics 106(4) (2000) 798-817.

[29] J.L. Johnson, K.R. White, J.E. Widen, J.S. Gravel, M. James, T. Kennalley, et al., A multicenter evaluation of how many infants with permanent hearing loss pass a two-stage otoacoustic emissions/automated auditory brainstem response newborn hearing screening protocol, Pediatrics 116 (3) (2005) 663-672.

[30] G.T. Mencher, A.C. Davis, S.J. DeVoe, D. Beresford, J.M. Bamford, Universal neonatal hearing screening: past, present, and future, Am. J. Audiol. 10 (2001) 3-12.

[31] K.R. White, T.R. Behrens, The Rhode Island Hearing Assessment Project: implications for universal infant hearing screening, Semin. Hear. 14 (1993) 1-119.

[32] National Institutes of Health, NIH Consensus Development Program, Early identification of hearing impairment in infants and young children, NIH Consens Statement 11 (1993) 1-24.

[33] Wessex Universal Neonatal Hearing Screening Trial Group (Kennedy CR et al.), Controlled trial of universal neonatal screening for early identification of permanent childhood hearing impairment, Lancet 352 (1998) 19571964.

[34] M.F. Hunter, L. Kimm, D. Cafarelli, C.R. Kennedy, A.R.D. Thornton, Feasibility of otoacoustic emission detection followed by $A B R$ as a universal neonatal screening test for hearing impairment, Br. J. Audiol. 28 (1994) 47-51.

[35] E.L. McNellis, A.J. Klein, Pass/fail rates for repeated clickevoked otoacoustic emission and auditory brain stem response screenings in newborns, Otolaryngol. Head Neck Surg. 116 (1997) 431-437.

[36] D.L. Stewart, A. Mehl, J.W. Hall III, V. Thomson, M. Carroll, J. Hamlett, Infant hearing impairment and universal hearing screening. Universal newborn hearing screening with automated auditory brainstem response: a multisite investigation, J. Perinatol. 20 (2000) S127-S130.

[37] C.J. Clemens, S.A. Davis, A.R. Bailey, The false-positive in universal newborn hearing screening, Pediatrics 106 (2000) e7 (Available at: http://www.pediatrics.org/cgi/content/ full/106/1/e7).

[38] J. Lemons, A. Fanaroff, E.J. Stewart, J.D. Bentkover, G. Murray, A. Diefendorf, Newborn hearing screening: costs of establishing a program, J. Perinatol. 22 (2002) 120-124.

[39] H.M. Yee-Arellano, F. Leal-Garza, K. Pauli-Müller, Universal newborn hearing screening in Mexico: results of the first 2 years, Int. J. Pediatr. Otorhinolaryngol. 70 (2006) 18631870.

[40] K.L. Iley, R.J. Addis, Infant hearing impairment and universal hearing screening. Impact of technology choice on service provision for universal newborn hearing screening within a busy district hospital, J. Perinatol. 20 (2000) S121S126.

[41] National Consortium for Newborn Hearing Screening, TEOAEBased Universal Newborn Hearing Screening, Georgetown 
A comparison of TEOAE and AABR for newborn hearing-screening

1201

University School of Medicine, Washington, DC, November 16-18, 1995.

[42] C.A. DeUzcategiu, C. Yoshinaga-Itano, Parents reactions to newborn hearing screening, Audiol. Today 24 (1997) 27.

[43] J. Sokol, M. Hyde, Hearing screening, Pediatr. Rev. 23 (2002) 155-162.

[44] E. Seifert, S. Hartmann, P. Weber, Otoacoustic emissions as indicators of neurologically based hearing loss in childhood, Neuropediatrics 28 (1997) 289-291.

[45] A. Majnemer, B. Rosenblatt, P. Riley, Prognostic significance of the auditory brainstem evoked response in high-risk neonates, Lev. Med. Child. Neurol. 30 (1998) 43-52.

[46] Z.D. Jiang, Maturation of peripheral and brainstem auditory function in the first year following perinatal asphyxia: a longitudinal study, J. Speech Lang. Hear. Res. 41 (1) (1998) 83-93.

[47] J.T. Jacobson, C.A. Jacobson, R.C. Spahr, Automated and conventional ABR screening techniques in high-risk infants, J. Am. Acad. Audial. 1 (4) (1990) 187-195.
[48] B.S. Herman, A.R. Thornton, J.M. Joseph, Automated infant hearing screening using the ABR: development and validation, Am. J. Audial. 4 (1995) 6-14.

[49] J.S. Gravel, K.R. White, J.L. Johnson, et al., A multisite study to examine the efficacy of the otoacoustic emission/ automated auditory brainstem response newborn hearing screening protocol: recommendations for policy, practice and research, Am. J. Audion. 14 (2) (2005) S217-S228.

[50] M.P. Gorga, K. Preissler, J. Simmons, L. Walker, B. Hoover Some issues relevant to establishing a universal newborn hearing screening program, J. Am. Acad. Audiol. 12 (2001) $101-112$.

[51] J.E. Widen, R.C. Folsom, B. Cone-Wesson, et al., Identificaion of neonatal hearing impairment: hearing status at 8 to 12 months corrected age using a visual reinforcement audio metry protocol, Ear Hear. 21 (2000) 471-487.

[52] M.P. Moeller, K.R. White, L. Shisler, Primary care physicians' knowledge, attitudes, and practices related to newborn hearing screening, Pediatrics 118 (4) (2006) 1357-1370.

Available online at www.sciencedirect.com

ScienceDirect 